\title{
A Petri Net Based System for the Modeling and Computer Simulation of Automated Construction Operations
}

\author{
Wakefield R.R* Damrianant J**, and O'Brien J.B* \\ School of Civil Engineering \\ The University of New South Wales, Sydney 2052, Australia \\ ** Senior Lecturers \\ r.wakefield@unsw.edu.au, j.obrien@unsw.edu.au \\ ** Doctoral Research Student,
}

\begin{abstract}
Simulation and modeling of construction systems has been the subject of much research over the past twenty years however there is still remains a need for a user friendly, high accuracy modelling method that comprehensively represents the complex dynamics and often time varying logics of the field construction process.
\end{abstract}

This paper seeks to illustrate how Petri nets, a powerful modeling tool initially developed for modeling computing process and networks and flexible manufacturing systems, can be successfully adapted to the modeling of automated construction systems, for the operational design of systems comprised of autonomous construction agents and robotic devices and for the quantitative modelling of flexible manufacturing processes.

Petri nets are generally considered to be superior for modelling systems with concurrency, where several state changes happen simultaneously and where event driven characteristics are present. The paper describes the Petri Net approach to modeling and shows how good dynamic models of automated construction systems can be developed. Several common Petri Net structures and features useful in construction system modeling are described. Time and colour Petri Nets and their application in modeling specific characteristics of construction systems are discussed. The paper concludes with a summary of the advantages and disadvantages of Petri nets as a tool for high accuracy computer modeling and simulation of automated and hybrid construction operations. Finally, some ongoing developments relative to the Petri net based construction simulation system are discussed.

\section{Introduction}

In the development of robotic and flexible manufacturing operations, modelling techniques that are considerably more sophisticated than those that are normally proposed for the modelling of construction operations [1] are required For example, in the development of a multiple-agent automated system [2] that could affect the erection of a simple stack-build falsework scaffold [3] or operate as a small fleet of automatically guided delivery vehicles (AGV's), modelling languages are required that can handle:

- Multiple, geographically dispersed, episodically operating work fronts.

- Discrete event form and continuous-event form field processes

- Parallel and serial production process logics.

- Conditionally variable, resource-allocation-to-task logics

- Absolute and conditional process start and/or finish times and in some case perishable goods types plus process sequence and synchrony constraints.

- Dynamic inter-agent role structure and time varying organisation.

- The operation of exogenous and endogenous variables

- Conflict between processes consequent upon task times taken and or/changed priorities.

- Resource and server queuing phenomena and processes.

- Floating resource pools and task-stacks.

- Multiple resource mobilisation and co-ordination logics prior to task commencement

- Policy based priority structures as to task or process performance.

- OH\&S operations constraints and (independent) safety manger operation.

- Interleaved disparate production processes.

- Gross methods switching in mid-stream. 
- Information flows and intelligence gathering activities either as resources, commodities or tasks

- Iterative and dynamic programming decision processes and interleaved ad-hoc (problem solving) activities.

- Flow of permissions and work-stage approvals.

- Rejections of parts of work as a result of inspections and work recycling plus treatment of errors and equipment breakdowns.

- Policy and strategy variables in construction task execution.

In the modelling of more complex activities such as are required for full automated building process modelling [4] there are the yet further requirements to be met. Thus, modelling of both on-site and off-site processes are required and a number of disparate technologies and production processes must be covered as interwoven processes and systems. Each of these interwoven processes is typically conceived of independently and managed independently but is coordinated with a central system or body of processes.

It follows from the above list that modelling languages that can both:

- model the logic of a fixed task and methods-

1. architecture and provide simulation methods for the dynamic execution of activities under internal and external parameter varying conditions.

2. permit dynamic variation of the overall method of work and role architectures (as meta-processes) over time.

are required if the full complexity of real-world systems are to be realistically dealt with.

A further requirement is that the modelling system in operation must be able to be used by practising managers and be at least somewhat userfriendly and economic.

\section{Available Modeling Languages And Procedures}

There are a number of modelling techniques that have been proposed as being suitable for construction process operations modelling $[5,6,1,7,8,9]$. Cyclone is a popular example of a construction simulation tool. Other simulation tools used for construction simulation include ithink $[10,11]$ and SLAM II $\{12,13]$. To the author's minds however none of the available methods is sufficiently versatile and flexible to meet the requirements of the above set of real-world model building criteria. One method however that does show promise in its ability to realistically and relatively simply model complex real world system is one based on the idea of Petri Nets. The Petri Net method has been successfully used in advanced manufacturing to model flexible manufacturing processes and within factory AGV operation [see eg. 14]. Petri Net modelling methods have the advantage of being able to handle multi-agent operations, flexible task and execution logics, co-ordination requirements and queuing processes.

The aim of this paper is to discuss the Petri-Net method in the context of construction robotics and automation and to illustrate its practical utility through the modelling a number of different types of civil engineering field operations.

\section{An introduction to Petri-Net modelling}

C. A. Petri introduced his networks in 1962. Since then many advances have been made in both theory and application. Petri Nets (PNs) have been developed by simulation researchers for general simulation applications. As a result, the names of the network components are simulation terms and may be unfamiliar to constructors. To ease the transition, a simple construction operation is used as an example.

Figure 1(a) is a graphical PN model of a crane hoisting materials from the ground to the workface. This network is made up of a number of connected symbols, each with the following attributes:

- 1. Circles (places) represent states of being. In construction, these are often states of readiness. The crane moves through the following states of readiness: crane ready to attach, ready to lift, ready to detach, and ready to return.

- 2. Squares (transitions) are actions which change the state of the system. Attach the load, lift and swing, detach the load, and crane return are transitions.

- 3. Black dots (tokens) are the resources of the operation. In this case the tokens are the materials being hoisted and the crane doing the hoisting. Pieces of information can also be taken as a resource.

- 4. Arrows (directed arcs) indicate the direction the resources (tokens) move when an action (transition) takes place. Transitions are said to "fire" as their action takes place.

The location of the materials and crane (tokens) in the network at any point in time is referred to as the "marking" of the net at that instant. In this example we start with two tokens, the crane and the materials to be lifted, both in their respective circles (places). At 
this point the transition "attach the load" is enabled and ready to fire. The firing of the transition moves the tokens from "crane ready" and "materials to be lifted" (the input places), and puts a single token in the place "ready to lift" (the output place). The result of this first transition firing is shown in Figure 1(b). Transitions continue to fire as they are enabled until the supply of tokens is exhausted.

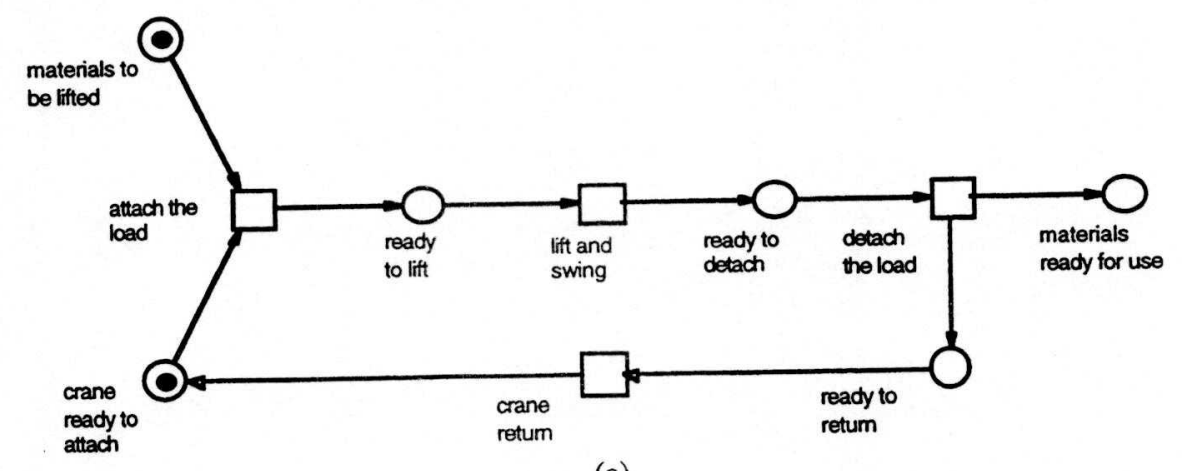

(a)

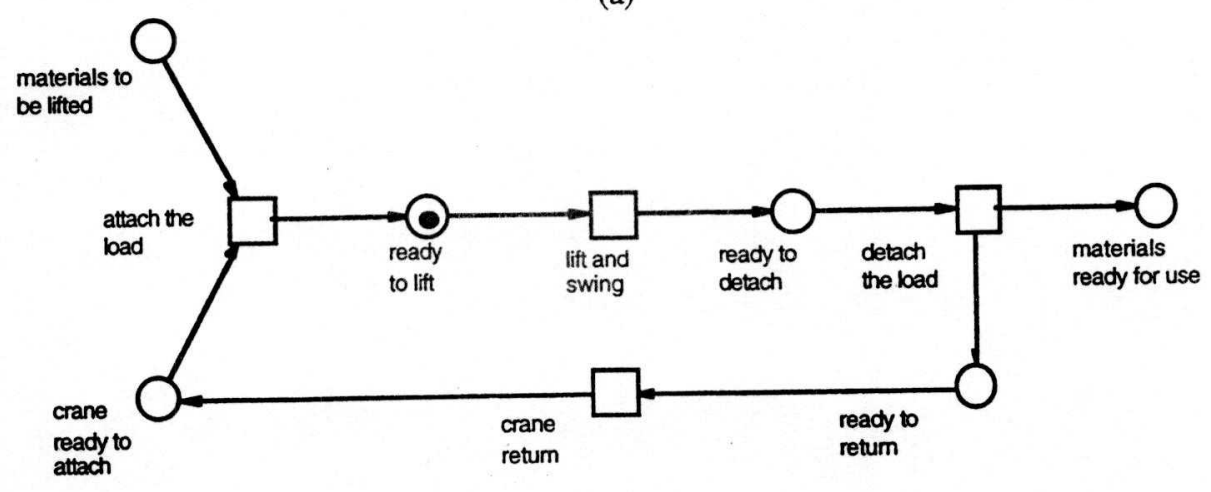

(b)

\section{Key}

Places - states of readiness

Transitions - actions that change the state of the system

- Tokens - equipment, resources, information Directed Ares - indicate the direction tokens move

Figure $1 \mathrm{~A}$ PN model of a crane hoisting materials

\section{1: Mathematical Definition}

In this section a basic introduction to PNs is given. The reader is referred to [15-17] for a more thorough treatment. A Petri net, in its most basic form, is a four tuple defined as:

$P N=\langle P, T, I N, O U T\rangle$ where

$P=\left\{p_{1}, p_{2}, p_{3}, \ldots, p_{n}\right\}$ the set of $n$ places
$T=\left\{t_{1}, t_{2}, t_{3}, \ldots, t_{m}\right\}$ the set of $m$ transitions

$I N=(P \times T) \rightarrow N$ is an input function which defines directed arcs from places to transitions OUT $=(P \times T) \rightarrow N$ is an output functon of directed arcs from transitions to places

The directed arcs can be weighted to indicate the number of tokens that are transferred to or from a place on the firing of a transition. 


\section{2: Properties of Petri Nets}

PNs exhibit a number of important properties which are useful in determining the behavior of the modeled system. These properties may prove useful in analyzing construction systems at the planning stage. A list of the properties, along with descriptions, are given below $[15,18]$ :

1. Safeness. A place is safe if the number of tokens in that place never exceeds one. A PN is safe if all its places are safe.

2. Boundedness. A place is $\mathbf{k}$-bounded if the number of tokens in that place cannot exceed $\mathrm{k}$.

3. Conservation. A net is strictly conservative if the number of tokens within a net remains constant. This concept is useful when tokens represent resources. Sometimes however, tokens represent counters or other non-resource items. Under these circumstances it might not matter if tokens are created or destroyed. To provide the benefit of conservatism without this problem, one can consider the net conservative only for tokens representing resources. This problem can also be overcome by using colored tokens.

4. Liveness. A transition is live if it is able to fire and is not deadlocked. If a transition is live it is not necessarily enabled but is potentially enabled under some attainable marking. This property can be used as a check on the logic of the system. If all transitions are live, given the correct conditions, they can fire and the state change represented by the transition can occur.

5. Reachability. Under the marking $\mu$, if it is possible to obtain the marking $\mu^{\prime}, \mu^{\prime}$ is said to be reachable. In Figure 1, the fact that the marking of one token in "ready to lift" is reachable from the initial marking means that the logic of the transition is correct.

These properties are not all applicable to all forms of PNs. The properties and associated techniques for their determination are fully treated by several researchers [e.g. 19,17].

Basic PNs are useful in investigating qualitative or logical properties of dynamic systems such as boundedness, and liveness.

\section{3: Petri Net Constructs Useful in Construction Process Modeling}

There are typical structures that are common in construction. In this section we identify several PN constructs [17] that represent particular characteristics of construction activities. The constructs are illustrated in Figure 2. A fuller treatment of the constructs is given in [20].

- (a) Sequential execution. Transition $t_{2}$ can fire only after the firing of $t_{l}$. This imposes a precedence constraint that is typical of some construction activities. The construct can also be used to model causal relationships between activities

- (b) Conflict. Transitions $t_{1}, t_{2}$, and $t_{3}$ are in conflict. All are enabled but firing of any leads to the others being disabled. The conflict can be resolved by assigning probabilities or priorities to the transitions. Performing one task stops the other task being undertaken at that time.

- (c) Concurrency. Transitions $t_{1}, t_{2}$ and $t_{3}$ are concurrent. This construct allows us to represent construction activities that proceed in parallel.

- (d) Synchronization. When several resources and pieces of equipment need to be available for an activity to proceed this type of construct can be used. The activity can proceed only when a token arrives in the place without a token, thereby synchronizing the firing of $t_{1}$ to the token arrival

- (e) Merging. This structure arises when several materials arrive for use at the same activity.

- (f) Confusion. This is the situation when conflict and concurrency exist together. In construction this sometimes occurs when resources are shared. It can be resolved by associating priorities or probabilities with the relevant transitions.

It is possible to build a logic model of most construction systems using a combination of these basic PN constructs. However, for quantitative modeling and analysis of construction systems the concepts of time and decision branching need to be added to basic PNs. 


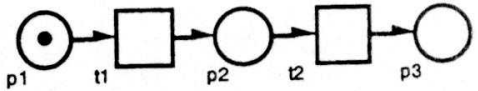

(a) Sequential execution
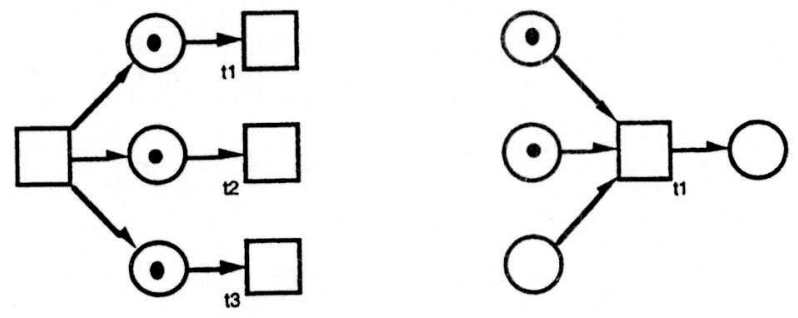

(c) Concurrency

(d) Synchronization

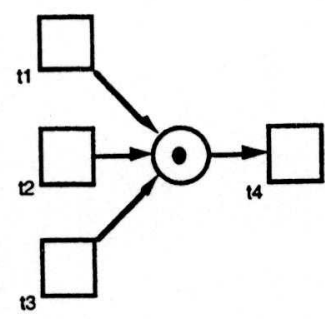

(e) Merging

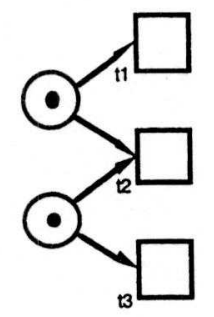

(f) Confusion

Figure 2 Typical PN constructs useful in construction modeling

\section{4: Time Petri Nets (TPN)}

To effectively model construction systems we need to be able to associate times with transitions (activities). This means that the transition will, when all the input conditions are met, take $t$ time units for the firing process to deposit tokens in the output places (for a full description of the development of TPNs [see:17,14,21,22]. The time delay can be deterministic or represented by any distribution function. It is also possible to associate time to other PN elements but in this work we will consider time to be associated only with transitions.

\section{5: Extended Transitions}

Extended transitions allow the modeler to put decision branches into the net. When tokens pass an extended

transition a decision must be made according to a given condition within the transition. The extensions to basic transitions that we use in construction modeling are probabilistic transitions and priority transitions. These extensions are necessary when two or more transitions are in conflict, that is, they both are enabled.

It is possible to give transitions a priority order for firing in such situations. When several transitions have the same priority and are in conflict, then it is possible to associate a probability with each which will determine which transition will fire first.

\section{6: Colored Petri Nets (CPNs)}

CPNs are extensions of PNs with identical modeling power but with increased graphical conciseness [18]. CPNs allow different attributes (colors) to be associated with tokens and for different colored tokens to be handled differently by the PN elements. For many modeling problems in construction it is necessary to distinguish between different information, material and resource flows. CPNs allow us to do this. Colored tokens are useful when several tasks need to share a scarce resource. For example, a crane lifting different materials on a construction site, each material can be represented by a different color token and handled differently by the crane. Materials, based on their "color" could be delivered to different processes or locations on the site. CPNs are also useful for modeling heterogeneous queuing situations in construction, in cases where equipment fleets are made up of different types of equipment (see for example [23]). In this situation non deterministic timed transitions can, if required, have different time distributions associated with different colored tokens.

\section{7: Queuing Disciplines for Places}

This extension to basic PNs allows queues of tokens that form at places to be governed by a queuing discipline. Such a facility is important in modeling some construction systems when resources are shared or where it is necessary to distinguish between queue members (if the queue members have different attributes) and process them accordingly. An example occurs in many earthmoving systems when first in first out queues are used for loading and different capacity trucks take different times to be loaded. 


\section{Example 1 Concrete Placement}

This example demonstrates that a PN based model produces very similar results to Microcyclone when used for simulating the same construction system. The concrete placement operation is described in detail in [1] and used as an example to demonstrate the capabilities of the Microcyclone system. Accordingly the same example is used here to show the reliability of Petri Nets on a classical construction simulation example. Basically, the example involves the dry batching of concrete materials into a truck at a batch plant location approximately two miles from the job site. Five batches are transported in each truck to the site where they are dumped individually and sequentially into the skip of a concrete mixer and mixed. After each batch is mixed it is dumped into a concrete bucket and lifted by crane to the placement location, where it is dumped spread vibrated and finished by a concrete placement crew.

The graphical PN model of the system is shown in Figure 3. The process begins with four trucks in the truck queue place. A truck leaves the queue and the 5 batch partitions in the truck are counted. A weighted arc places 5 counter tokens (each representing a batch in the following place). When a batch is available the truck partition is loaded. This process is repeated 5 times until the truck is fully loaded. The fully loaded condition is represented by a marking of 5 on the place 'truck loaded'. The truck then begins the haul cycle. On reaching the mixer, providing the mixer is available, the batches are dumped sequentially into the mixer. At the completion of the mixing the concrete is placed in a one of two buckets for lifting to the work site. When the crane is available it lifts the bucket to the worksite for placement by the concrete placement crew.

The Visual Simnet program [23] was used to run the numerical model of the PN. The resources used in the simulation and the duration of the activities are identical to those used by [1]. One hundred cycles of dumping and placement of concrete were simulated using both Microcyclone and the PN numerical model. The time for one hundred cycles was 569.75 minutes using both Petri Net and Microcyclone. Results obtained indicate good agreement within the limits of numerical accuracy reported by each program. Full details of the results are given in [20] This example shows that Petri nets can reliably model construction processes and produce similar results to Microcyclone.

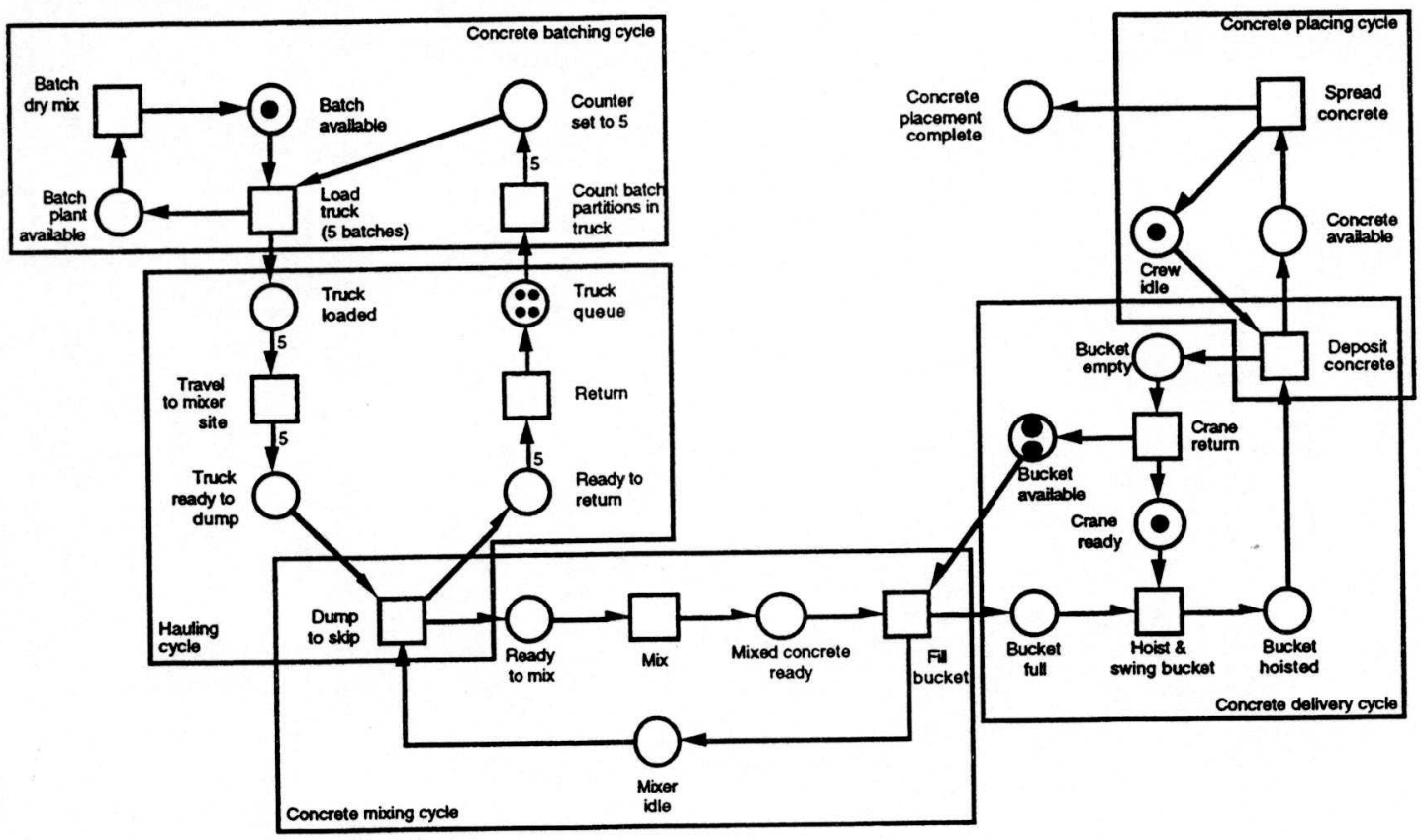

Figure 3 PN model of a concrete placing operation 


\section{Example 2 Hong Kong Airport Earthmoving}

This second example illustrates several powerful features of Petri Nets not available in other construction simulation programs. This example makes use of colored tokens, probabilistic transitions and queuing priorities, in modeling an earthmoving system.

The system modeled here is loosely based on a sub-system of the earthwork construction system used in a civil works contract for the New Hong Kong International Airport. The main aim of the example is to demonstrate the capability of extended PN models in representing complex construction systems.

The initial stage of the civil works contract involved the expansion of a small existing island by dredging and land based earthmoving, into a 1248 hectare island, $4.5 \mathrm{~km}$ end to end and $3.5 \mathrm{~km}$ at its widest point. The fill for the land based part of the contract was provided by leveling the existing island and importing excavated material from other parts of Hong Kong. The land based part of the contract was one of the world's largest earthmoving operations involving approximately 100 million cubic meters of material in 31 months [25]. As part of the contract the Provisional Airport Authority (the client) supplied approximately one third of the equipment needed for the earthmoving operations. This included four Demag H285 loaders, seventeen trucks (Cat 785) and other drill and blast equipment. The civil works contract was split into two subcontracts. The supplied equipment was divided between the two subcontractors. The subcontractors then added to the supplied equipment to meet their operational requirements. One subcontractor did this with a mixed fleet of trucks, including Cat 777 and Cat 785 trucks, while the other chose to use a fleet of Cat 785 trucks. [25] gives a description of the systems used. The subcontractors also used different loader truck combinations and operating policies. There are a number of aspects of the operation that could be investigated using a Petri Net simulation including economic analysis of different truck loader combinations and the effect on production of different operating policies, however this is beyond the scope of the present paper. For the purposes of this paper we will model a one loader truck fleet combination that was utilised as part of the earthmoving process.

The objective of this example is to demonstrate that PNs can usefully model earthmoving systems and to compare the production performance of a mixed truck fleet and a homogeneous truck fleet and . The method of excavation used at the New Hong Kong Airport site was drill and blast. Material was then loaded into trucks using excavators. Based on the quality of the excavated material it was hauled to one of three fill locations, A, B and C. Generally, trucks of two capacities were used on the site: Cat 777 trucks with capacity 30,33 and $35 \mathrm{~m}^{3}$ of material types A, B, and $\mathrm{C}$, respectively; and Cat 785 trucks with capacity 50, 55 and $60 \mathrm{~m}^{3}$ of material types $\mathrm{A}, \mathrm{B}$ and $\mathrm{C}$, respectively. The loader used in this example is a Demag H285 with an $18 \mathrm{~m}^{3}$ rock bucket. The loader required two to three bucket loads to fill the Cat 777 trucks and three to four bucket loads to fill the Cat 785 trucks. The PN model of the load, haul, dump operation is shown in Figure 5. To model some of the important characteristics of the construction operation it was necessary to include the following aspects in the PN model:

- a) Different types of trucks needed to be allowed to work together in a truck fleet. The model needed to include different characteristics of the trucks including capacities and performance characteristics.

- b) Three separate load destinations had to be available for the excavated material. The probabilities of loading each type of material had to be included in the model.

- c) The model needed to include the possibility of breakdown of trucks on both the haul and return legs of the operation.

- d) Non deterministic times for loading hauling and returning.

Inclusion of these aspects requires colored tokens to represent the different truck models. In the example we have included two types of trucks but it is possible to model each truck individually if required. Breakdown probabilities are associated with each type of truck to model the situation in which one type of truck is more reliable than another. First in first out queuing is used at the loader, the dump site and when repairing breakdowns. Full details of the example including input parameters and results are given in [20]. Durations of transitions are based on information obtained from the subcontractors and collected on site.

The simulation was carried out using three different truck fleet combinations. Daily production averages are based on two 11 hour shifts per day. Transient effects are ignored in this analysis. The results indicate that roughly equivalent daily production can be obtained with each of the truck fleets modelled. This example indicates that the Petri Net model can provide production rates, cycle times and equipment utilisation information that with production costs and economic information regarding the equipment could be used by contractors to determine the optimum 
earthmoving system. The extended PN model of the process effectively represents the earthmoving process and handles the heterogenous queues, non deterministic transitions, different load destinations and breakdown probabilities.

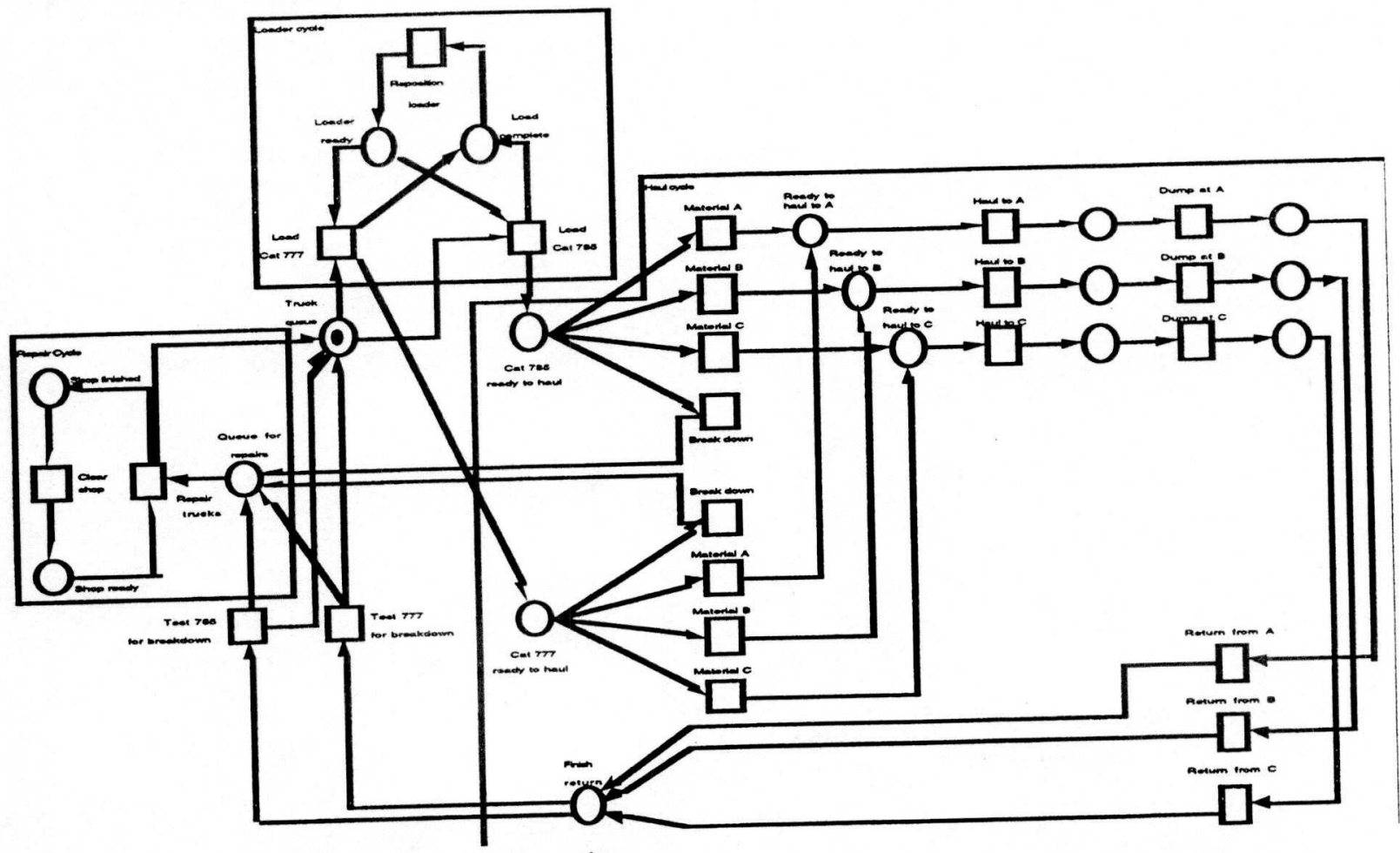

Figure 4 PN model of the earthmoving operation

\section{Discussion}

These examples demonstrate how PN models can be effectively used for both qualitative and quantitative modeling and analysis of construction operations for the purpose of automation development. The graphical nature of the models make them relatively easy to understand and to communicate to others and their ease of conversion into algebraic form means analysis is straightforward. Petri Nets are capable of modeling phenomena present in construction operations including non deterministic activity times, attaching priorities to particular activities, probabilistic branching and queueing disciplines. The use of colored Petri Nets, demonstrated in Example 2, gives further capability for the user to differentiate between different types of equipment, information and resource flows. In Example 2, the colored PNs allow the simulation model to account for the different truck capacities they could also allow for different loader characteristics or different repair shop performance if required. This feature is of particular use when modeling more complex construction operations. The other feature of the Petri Net modelling system which it is not possible to demonstrate in this paper is the ability to animate the graphical model of the simulation. This feature is useful when debugging the simulation model and also in improving the construction process design. The modeling power of Petri Nets when combined with their simplicity make them a powerful and accessible tool for construction engineers for the modeling and simulation of automated construction systems.

The disadvantages with PNs in the construction context include the terminology of the modeling system and the fact that most software tools are not aimed at construction users. Potential improvements could include provision of a construction terminology based interface. Another improvement to the enhanced stochastic PN modeling system presented in this paper would involve making transition behavior variable and dependent on token attributes and elapsed time. For example in the earthmoving system described above, breakdown probabilities could be related not only to the type of truck but to the number of hours of operation since last overhaul. Travel times could also be varied with the truck or road conditions. Work on these enhancements to the present system is underway. 


\section{Conclusions}

Petri-Nets are a reasonably straight-forward and versatile way of modelling complex-logic factory and field activities. With suitable extensions, this method may prove most useful in the high-fidelity modelling of field robot activities and automated construction operations.

\section{References}

1. Halpin, D. W. and Riggs, L. S. (1992). Planning and Analysis of Construction Operations, John Wiley and Sons, Inc. New York, N.Y.

2. Khatib, O. "Mobile robotic manipulation" 26th ISIR Singapore, October 4-6 1995 pp 7-12. 1996

3. UNSW 4th year Civil engineering undergraduate students reports "Experiments in the erection of PAL scaffolding under laboratory conditions". Subject Civl 8.0181990

4. O'Brien, J.B “Automated production of genericform reinforced concrete structures through use of an improved progressive strength construction method" 14th ISARC Pittsburgh, 1997

5. Halpin, D. W. (1977). "CYCLONE : method for modeling of job site processes." J. Constr. Div., ASCE, 103 (3), 489-499.

6. Pilcher, R. and Flood, I. (1984). "The use of simulation models in construction." Proc. Instn. Civ. Engrs., Part 1(76), 635-652

7. Lutz, J. D. and Hijazi, A. (1993). "Planning repetitive construction: current practice." Construction Management and Economics, 11, 99-110.

8. Vanegas, J. A., Bravo, E. B. and Halpin, D. W. (1993). "Simulation technologies for planning heavy construction processes." J. Constr. Engrg. and Mgmt., ASCE, 119 (2), 336-356.

9. Farid, F. and Koning, T. L. (1994). "Simulation verifies queuing program for selecting loader-truck fleets." J. Constr. Engrg. and Mgmt., ASCE, $120(2), 386-404$

10. Senogles, J. C. and Peck, G. M. (1994) "Application of computer simulation techniques to evaluation of environmental impacts on underground construction for the Sydney Harbour Tunnel" in Wakefield, R.R. and Carmichael, D.G. (eds), Construction and Management: Recent Advances. A.A. Balkema, Rotterdam, 305-316

11. High Performance Systems Inc.(1994). ithink User's Manual, Hanover, N.H.

12. Gonzalez-Quevedo, A. A., AbouRizk, S. M., Isley, D. T. and Halpin, D. W. (1993)
"Comparison of two simulation methodologies in construction" J. Constr. Engrg. and Mgmt., ASCE, 119 (3), 573-589.

13. Pritsker, A. A. B. (1986) Introduction to simulation and SLAM II, John Wiley, New York.

14. Ravi Raju, K. and Krishnaiah Chetty, O. V. (1993). "Design and evaluation of automated guided vehicle systems for flexible manufacturing systems: an extended timed Petri net-based approach.” Int. J. Prod. Res., 31 (5), 1069-1096.

15. Peterson, J. L. (1981). Petri Net Theory and the Modeling of Systems. Prentice Hall Inc., Englewood Cliffs, New Jersey.

16. Resig, W. (1985) Petri Nets: An Introduction, EACTS Monographs on Theoretical Computer Science, Springer-Verlag, Berlin.

17. Viswanadham, N. and Narahari, Y. (1992). Performance Modeling of Automated Manufacturing Systems. Prentice Hall Inc., Englewood Cliffs, New Jersey.

18. Cossins, R. and Ferreira, P. (1992). "Celeritas: a colored Petri net approach to simulation and control of flexible manufacturing systems." International Journal of Production Research, 30 (8), 1925-1956.

19. Viswanadham, N. and Narahari, Y. (1987). "Colored Petri net models for automated manufacturing systems." IEEE International Conference on Robotics and Automation, 19851990.

20. Wakefield, R. R and Sears, G. A, "Petri Nets for simulation and modelling of construction systems" to appear, ASCE Journal of Construction Engineering and Management, June 1997.

21. Venkatesh, K., Kaighobadi, M., Zhou, M.C. and Caudill, R. J. (1994). “Augmented timed Petri nets for modeling, simulation, and analysis of robotic systems with breakdowns." Journal of Manufacturing Systems, 13 (4), 289-301.

22. Lin, J. T. and Lee, C. C. (1994). "Modular modeling for performance evaluation of robot centered manufacturing cells using timed Petri nets." Int. J. Adv. Manuf. Technol., 9, 271-280.

23. McCahill, D. F. and Bernhold, L. E. (1993). "Resource-oriented modeling and simulation in construction." J. Constr. Engrg. and Mgmt., ASCE, 119 (3), 590-606.

24. Garbe, W. (1995). Visual Simnet - Users Manual, unpublished, Dresden.

25. Allwood, P. (1993). "Earthworks off to a flying start." in Hong Kong's Replacement Airport at Chep Lap Kok, Joem Publishing, Hong Kong. 